\title{
Coronary microvascular dysfunction and flow reserve: an update
}

\author{
Paolo G. Camici · Ornella E. Rimoldi
}

Received: 16 September 2013/Accepted: 8 October 2013/Published online: 18 October 2013

(C) Italian Association of Nuclear Medicine and Molecular Imaging 2013

\begin{abstract}
Non-invasive imaging techniques (positron emission tomography and cardiovascular/cardiac magnetic resonance) provide accurate and reproducible measurements of global and regional myocardial blood flow (MBF) in absolute units of $\mathrm{ml} / \mathrm{min} / \mathrm{g}$ of tissue, thus providing unique pathophysiological and diagnostic insights into the functioning of the coronary microcirculation. There is compelling evidence suggesting that, in many instances, in the absence of angiographically demonstrable stenosis of the epicardial coronary arteries, significant abnormalities of global myocardial perfusion can be demonstrated in individuals with either risk factors for coronary artery disease or different cardiomyopathies accompanied by alteration of the structure of the microvasculature. In this context, measurement of MBF gives unique diagnostic information regarding the functioning of the coronary microcirculation and provides a quantitative surrogate endpoint against which the efficacy of treatments can be established. In this review article, we discuss the more recent advances regarding the non-invasive measurement of MBF and flow reserve and their clinical applications for the detection of coronary microvascular dysfunction.
\end{abstract}

Color figures online at http://link.springer.com/article/10.1007/ s40336-013-0037-8.

P. G. Camici

San Raffaele Scientific Institute, Vita Salute University,

Milan, Italy

O. E. Rimoldi $(\square)$

C.N.R., Istituto di Bioimmagini e Fisiologia Molecolare, Centro PET, Istituto Scientifico Universitario San Raffaele, Settore C, piano -2, Via Olgettina, 60, 20132 Milan, Italy

e-mail: rimoldi.ornella@hsr.it
Keywords Microcirculation - Myocardial perfusion $\cdot$ PET $\cdot$ CMR

\section{Background}

Obstructive disease of the epicardial coronary arteries has been recognized as the cause of myocardial ischemia and angina pectoris for more than two centuries. Therefore, these arteries have been the focus of both diagnostic and therapeutic attention. In the late 1950s, Sones introduced coronary angiography, which provided a "road map" of the epicardial coronary arterial tree $[1,2]$. This was followed by the development, first of coronary artery bypass grafting, in 1970 [3], and then of percutaneous coronary angioplasty, in 1977 [4].

In the past three decades, however, several studies have shown that abnormalities in the coronary microcirculation may also cause or contribute to myocardial ischemia in several conditions. It has been suggested that in a number of patients admitted with angina attacks but no apparent cardiac or systemic disease, coronary microvascular dysfunction (CMD) could represent the sole cause of the symptoms [5]. Myocardial blood flow (MBF) abnormalities and the consequent CMD-related myocardial alterations may differ substantially from those caused by flow-limiting stenoses in epicardial coronary arteries. In the latter case, the impairment of MBF is generally regional and distributed within the territories subtended by the stenosed artery, resulting in detectable segmental impairment of contractile function. In the case of CMD, on the other hand, the abnormality may not necessarily involve the territory subtended by a major coronary branch; indeed, it may involve the whole left ventricle (LV) diffusely or be distributed in a scattered manner [5]. 
Camici and Crea [5] have proposed a clinical classification of CMD, dividing it into four main types on the basis of the clinical setting in which it occurs [1]: (1) CMD in the absence of myocardial diseases and obstructive coronary artery disease (CAD), (2) CMD in myocardial diseases, (3) CMD in obstructive CAD, and (4) iatrogenic CMD.

CMD may be underlain by several pathogenetic mechanisms including structural, functional and extravascular alterations that can contribute to the condition in different ways. The importance of these mechanisms varies in different clinical conditions, although several of them may coexist in a single condition.

\section{Structural alterations}

Structural abnormalities responsible for CMD have, in particular, been consistently documented in patients with hypertrophic cardiomyopathy (HCM) and in those with arterial hypertension. In both these conditions, morphological changes are characterized by an adverse remodeling of intramural coronary arterioles consisting of vessel wall thickening, due mainly to hypertrophy of smooth muscle cells and increased collagen deposition in the tunica media, with variable degrees of intimal thickening $[2,5]$. This thickening of the vascular wall leads to an increase in the medial wall area, and a relative reduction of the size of the lumen that correlates with the increase in minimal coronary resistance and the reduction of coronary flow reserve (CFR). Although qualitatively similar in the two conditions, these anatomical changes are usually quantitatively more severe in patients with HCM.

An important feature shared by patients with arterial hypertension and those with $\mathrm{HCM}$ is the diffuse nature of the microvascular remodeling, which involves the entire LV independently of the distribution of the ventricular hypertrophy (i.e., symmetrical vs asymmetrical), and may also involve portions of the right ventricle [6]. The functional counterpart of this phenomenon is the maximum MBF and CFR blunting in the whole LV that has been demonstrated in most of these patients [7]. Interestingly, similar changes have been described in the spontaneously hypertensive rat, which is, therefore, a good model of coronary microvascular remodeling associated with hypertension $[8,9]$.

Functional alterations

Functional CMD may be caused by a variable combination of mechanisms leading to impaired coronary microvascular dilatation and mechanisms resulting in increased microvascular constriction.
Endothelial cells play a major role in the regulation of blood flow in the coronary microcirculation, particularly at the level of the pre-arteriolar vessels, both in resting conditions and during increased myocardial oxygen consumption. Accordingly, alterations in endothelial function may impair MBF both at rest and in the event of an increased myocardial workload, as typically seen during exercise [10].

Impairment of endothelium-independent dilatation has been shown to be a cause of CMD in several experimental and clinical conditions characterized by a significant reduction of the MBF increase in response to direct arteriolar/pre-arteriolar vasodilators (e.g., adenosine, dipyridamole, papaverine). Despite the large amount of data documenting the role of endothelium-independent dilatation in CMD, the cellular mechanisms involved remain incompletely understood $[10,11]$.

Evidence to support the notion that coronary microvascular constriction may cause myocardial ischemia has been found both in experimental models and in man. Some vasoconstrictor substances, indeed, cause selective intense microvascular constriction with minimal effects on the epicardial coronary arteries [11].

\section{Extravascular mechanisms}

The pulsatile pattern of blood flow shows typical physiological variations, linked to the variations in intramyocardial and intraventricular pressure occurring during the systolic and diastolic phases of the cardiac cycle. MBF mainly occurs in diastole and, therefore, diastolic abnormalities particularly affect myocardial perfusion. Nevertheless, increased systolic intramyocardial and ventricular pressure, as typically seen in primary and secondary left ventricular hypertrophy, can also negatively impact on myocardial perfusion [12]. Indeed, an increase in microvascular compression generated during contraction makes subendocardial vessels less able to promptly regain their tone in diastole, a circumstance that results mainly in diastolic impairment of microvascular blood flow in subendocardial layers [13]. Diastolic blood flow is impaired by all conditions that increase intraventricular diastolic pressure. Thus, it is impaired in the presence of left ventricular hypertrophy and also in the presence of diastolic dysfunction related to increased collagen content in the myocardial tissue. Of note, conditions that make arteriolar driving pressure during diastole significantly lower than intraventricular pressure, such as aortic stenosis, critical coronary stenoses, prearteriolar constriction or merely hypotension, may facilitate diastolic impairment of MBF in this setting. The detrimental effects of increased systolic intramyocardial pressure and of diastolic intraventricular 
Fig. 1 In the absence of coronary stenosis (in the right panel angiography shows a patent left coronary artery) maximum $\mathrm{MBF}$ and $\mathrm{CFR}$ (maximum MBF/Rest MBF) reduction during adenosine infusion reflects the impairment of microvascular function (color figure online)

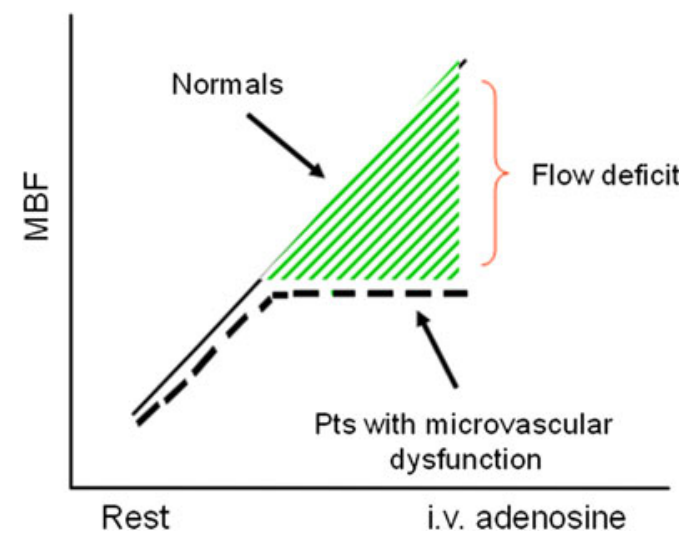

pressure on MBF are more pronounced in subendocardial than in subepicardial layers.

\section{Methods for studying coronary microvascular function}

Unlike epicardial coronary arteries, the coronary microcirculation cannot be directly imaged in vivo either with coronary angiography or intracoronary imaging techniques. Indeed, small coronary arteries are below the spatial resolution of coronary angiography (about $0.5 \mathrm{~mm}$ ) and cannot be reached by the catheters utilized for intravascular imaging or optical coherence tomography.

Assessment of coronary microvascular function, therefore, relies on the measurement of parameters that reflect its functional status, such as MBF and CFR. This latter is the ratio of MBF during pharmacologically induced arteriolar vasodilatation, achieved by means of intravenous or intracoronary vasodilators, to baseline resting MBF. Adenosine, because of its safety profile, is the vasodilator most widely used to assess hyperemic blood flow. However, some limitations must be taken into consideration. When it is administered systemically, hypotension and reflex tachycardia alter the coronary blood flow response and coronary vasomotor tone mediated by $\alpha$-receptors is not fully eliminated resulting in "near" maximal vasodilatation [14].

CFR is an integrated measure of flow through both the large epicardial coronary arteries and the microcirculation [15]. In the absence of obstructive stenoses of the epicardial arteries, a reduced CFR is a marker of CMD (Fig. 1). Although a single cutoff value of CFR (e.g., $\leq 2.0$ ) below which microvascular function is deemed abnormal would be useful clinically, it must be noted that, in normal humans, CFR varies according to age and gender [16]. Therefore, it is essential to compare CFR data obtained in patients with those obtained in age- and sex-matched normal subjects. In addition, resting MBF is linearly related to cardiac workload. Therefore, when comparing different patients, it is important to correct resting MBF for the main determinants of external cardiac workload, i.e., blood pressure and heart rate (rate-pressure product; RPP). A corrected CFR can then be calculated by dividing hyperemic flow by RPP-corrected resting flow [17-19]. More complex, on the other hand, is the assessment of CMD in territories subtended by stenotic coronary arteries, where the evaluation of microvascular function depends on the clinical context.

Hajjiri et al. [20] proposed that maximum MBF (i.e., the flow measured during pharmacological vasodilatation) might be superior to CFR. Their data are consistent with previous reports indicating that the degree of impairment of MBF flow bears important prognostic information [21]. In fact, it has been demonstrated that both in hypertrophic [22] and dilated [23] cardiomyopathies, the severity of impairment of peak MBF measured during dipyridamole stress is predictive of major adverse cardiac events at follow-up. In patients affected by these conditions, in whom there is no overt evidence of $\mathrm{CAD}$, the curtailment of $\mathrm{MBF}$ is due to dysfunction of the coronary microcirculation. This could be explained, at least in part, by the fact that CFR is a ratio and factors that influence either the numerator or the denominator may affect its calculation. Therefore, a reduced CFR does not necessarily reflect a reduction of maximum flow, but may be due to an abnormally elevated resting flow in the face of a normal hyperemic flow. This problem can be overcome, at least in part, by normalizing resting flow for the external cardiac workload [17, 18].

Although much of the excess of CAD risk can be accounted for by the presence of diabetes-associated coronary risk factors such as obesity, dyslipidemia and hypertension, a significant proportion of it remains unexplained. A direct deleterious effect of diabetes on vascular and, in particular, endothelial function has been suggested, which would increase the potential for vasoconstriction and thrombosis. There is consistent evidence that CFR is impaired in patients with diabetes and that this may be an early marker of atherosclerosis. A positron emission 
tomography (PET) study found marked CMD in response to adenosine (reflecting primarily endothelium-independent vasodilatation) and a cold pressure test (reflecting primarily endothelium-dependent vasodilatation) in young subjects with uncomplicated diabetes. The findings were very similar in type 1 and type 2 diabetes [24]. This provides further support for a key role of hyperglycemia in the pathogenesis of vascular dysfunction in diabetes [5].

\section{Positron emission tomography}

PET has been shown to allow non-invasive and accurate quantification of regional $\mathrm{MBF}$, providing suitable tracers are used and appropriate mathematical models applied. These PET measurements of MBF, for which the symbol $F / W$ is also used, are expressed as units of volume per time per unit weight of myocardium (i.e., $\mathrm{ml} / \mathrm{min} / \mathrm{g}$ ) $[15,19]$.

Different tracers can be used for measuring MBF using PET, including oxygen-15-labeled water $\left(\mathrm{H}_{2}^{15} \mathrm{O}\right)$ [25-27], ${ }^{13} \mathrm{NH}_{3}$ [28-31] and the cationic potassium analog rubidium-82 $\left({ }^{82} \mathrm{Rb}\right)[32,33] .{ }^{13} \mathrm{NH}_{3}$ and ${ }^{82} \mathrm{Rb}$ are given intravenously as boluses. In the case of $\mathrm{H}_{2}^{15} \mathrm{O}$, the tracer can be administered as an intravenous bolus injection [34-36] or an intravenous slow infusion $[36,37] .{ }^{82} \mathrm{Rb}$ is produced by a Strontium- $82 /{ }^{82} \mathrm{Rb}$ generator that generally needs replacing once a month. ${ }^{82} \mathrm{Rb}$ is a very appealing $\mathrm{MBF}$ tracer because it does not require a cyclotron on site and has a very short $t_{1 / 2}(78 \mathrm{~s})$ [33]. The high positron energy of ${ }^{82} \mathrm{Rb}$ results in a relatively inferior image quality and reduced spatial resolution due to its long positron track (4 mm). Kinetic models have been proposed for quantification of $\mathrm{MBF}$ using ${ }^{82} \mathrm{Rb}$ [38-40]; however, these are limited by the dependence of myocardial extraction of this tracer on the prevailing flow rate and myocardial metabolic state. Nevertheless, in the past few years, both semiquantitative [41] and fully quantitative measurements [42] of myocardial perfusion with ${ }^{82} \mathrm{Rba}$ have proven feasible on a large scale and provide incremental prognostic information both in patients with suspected and in those with known CAD.

Because of its ability to provide non-invasive regional absolute quantification of MBF, PET has been widely used to assess CFR in healthy volunteers. Chareonthaitawee et al. [16] investigated the range of resting and hyperemic MBF in a large population $(n=160)$ of healthy males and females with a broad age range (21-86 years). They found baseline and hyperemic MBF to be heterogeneous both within and between individuals. Furthermore, baseline and hyperemic MBF exhibited a similar degree of spatial heterogeneity, which appeared to be temporally stable. Resting myocardial perfusion ranged from 0.59 to $2.05 \mathrm{ml} / \mathrm{min} /$ $\mathrm{g}$ (average $0.98 \pm 0.23 \mathrm{ml} / \mathrm{min} / \mathrm{g}$ ) and adenosine-induced hyperemic perfusion ranged from 1.85 to $5.99 \mathrm{ml} / \mathrm{min} / \mathrm{g}$ (average $3.77 \pm 0.85 \mathrm{ml} / \mathrm{min} / \mathrm{g}$ ). Significant differences within subjects were found when comparing different segments with each other, except for anterior versus lateral regions. MBF was significantly higher in females than in males. A significant linear association was found between age and baseline MBF, partly related to changes in external cardiac workload with age. Hyperemic MBF declined after the age of 65 years.

Different studies have tested the short-term reproducibility of MBF measurements using PET with ${ }^{13} \mathrm{NH}_{3}, \mathrm{H}_{2}^{15} \mathrm{O}$ and ${ }^{82} \mathrm{Rb}$ [27, 43, 44]. Repeated measurements of resting and hyperemic MBF using intravenous dipyridamole and adenosine during the same study session were not significantly different, demonstrating the validity of the technique. The variability of hyperemic flow was larger, as indicated by a greater repeatability coefficient, and was paralleled by a greater variability of the RPP. This could mean that the greater variability of MBF during stress is more likely due to a variable response to vasodilators rather than to a larger measurement error. The feasibility and reproducibility of MBF measurement during supine bicycle exercise have been tested as well. The study results demonstrated the feasibility of this protocol, which was found to be at least as repeatable as using adenosine stress [45]. More recently, Jagathesan et al. [46], using PET with $\mathrm{H}_{2}^{15} \mathrm{O}$, tested the long-term reproducibility of MBF measurement at rest and following dobutamine stress in patients with stable CAD. Dobutamine induced reproducible changes in both global and regional MBF and flow reserve over a time interval of 24 weeks. The reproducibility of MBF and CFR with dobutamine was comparable with the short-term repeatability reported for adenosine and physical exercise in healthy subjects.

\section{Clinical perspective}

Myocardial perfusion imaging and CFR have a predictive value with regard to long-term prognosis in patients with suspected myocardial ischemia. In patients with normal perfusion, abnormal CFR is independently associated with a higher annual event rate over 3 years compared with normal CFR [21] (Fig. 2). A hybrid multislice CT system can provide comprehensive assessment of cardiac and coronary morphology in addition to assessment of inducible ischemia [42] in suspected CAD [47]. Both the coronary calcium content and the presence of coronary vascular dysfunction are associated with an increased risk of adverse cardiac events. However, in a large cohort of 901 patients, Naya et al. [48] found that in symptomatic patients without obstructive CAD, after adjustment for clinical risk, only coronary vascular dysfunction improved 


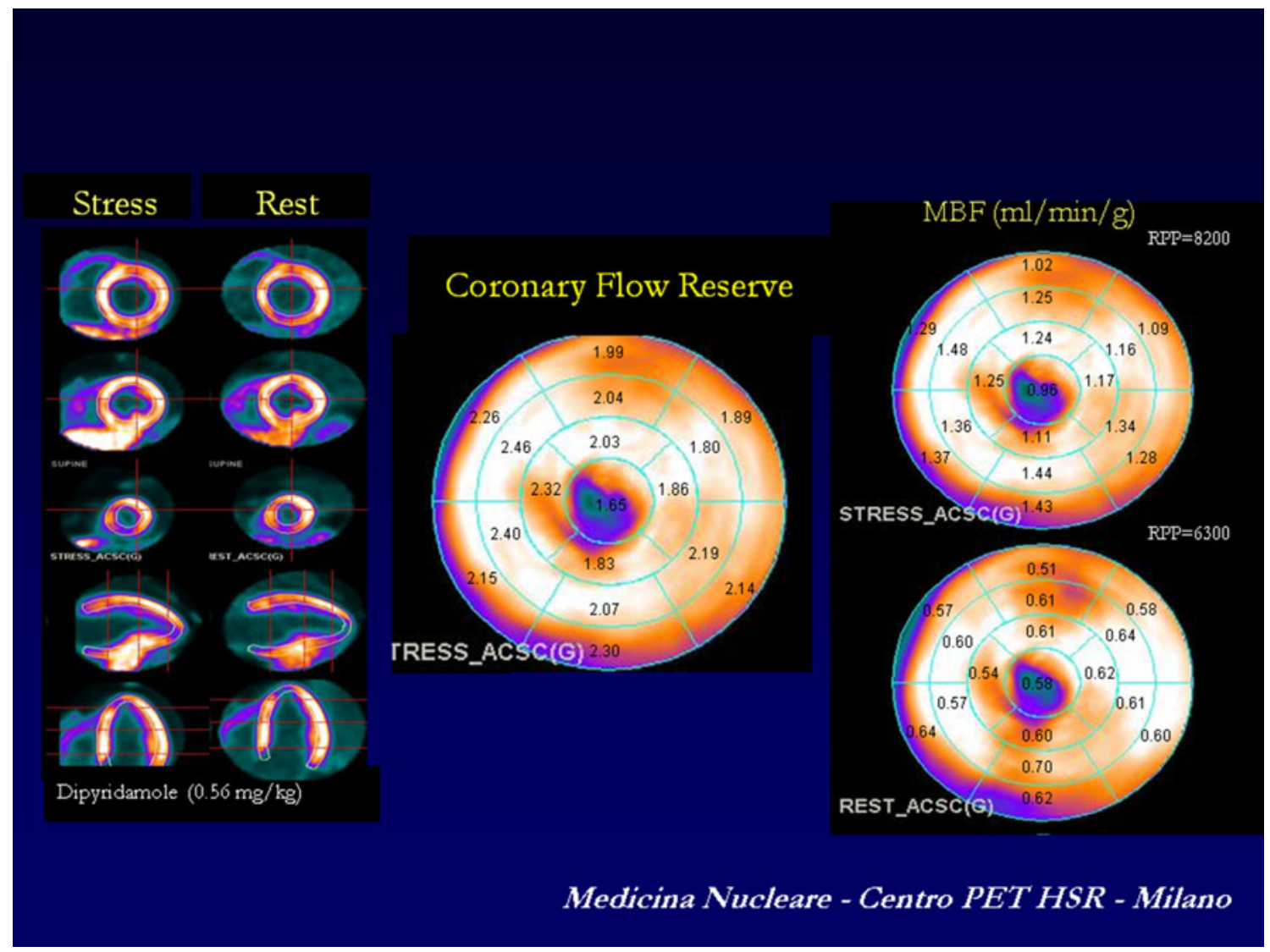

Fig. 2 Left panel PET scan with ${ }^{13} \mathrm{NH}_{3}$ in a young patient showing a perfusion defect in the apex, but apparently normal flow in the rest of the myocardium. Quantification of absolute myocardial blood flow (right panel) and flow reserve (middle panel) demonstrated diffuse

risk assessment, thus confirming that the total burden of coronary calcium deposits is only modestly associated with impaired vascular function.

PET is particularly effective in those circumstances in which the CFR is diffusely (and not regionally) blunted, e.g., in patients with balanced ischemia [49] and with hypertrophic or dilated cardiomyopathy [7].

In primary cardiomyopathies, postmortem studies on patients who died suddenly, or at transplant have provided compelling evidence for the occurrence of myocardial ischemia in HCM, despite normal coronary angiograms, showing frequent and often extensive areas of myocardial damage exhibiting all stages of ischemic injury [50]. Myocardial ischemia is often silent in HCM patients, and standard techniques employed over the years to assess hypoperfusion or ischemia, such as standard exercise testing, stress echocardiography and thallium-201 scintigraphy, have proven neither sensitive nor specific in this disease [51]. Our group, using PET, first demonstrated the occurrence of severe CMD in HCM patients not only in the hypertrophied septum, but also in the non-hypertrophied LV free wall. Subsequent studies using PET and, more recently, impairment of hyperemic (dipyridamole stress) myocardial blood flow and flow reserve. Courtesy of Dr. L. Gianolli, Dr. V. Bettinardi and Dr. E. Busnardo (color figure online)

cardiovascular/cardiac magnetic resonance (CMR), have confirmed that CMD is a diffuse phenomenon in HCM hearts $[7,11,51]$. Nevertheless, the absolute degree of microvascular impairment remains partly related to the extent of LVH, with the most severe blunting generally occurring at the septal level, where maximum wall thickening is usually present [51].

In patients with systemic hypertension, myocyte hypertrophy is accompanied by structural and functional alterations of coronary arterioles [2, 52], whereas larger intramyocardial arterioles do not show significant wall thickening. The reduction of CFR in hypertrophied hypertensive hearts is caused both by a concomitant increase in resting MBF, due to increased workload and oxygen consumption, and by a reduction of the hyperemic response to endothelial-dependent [53, 54] and independent stressors [53]. Interestingly, in a pre-clinical model, spontaneously hypertensive rats treated with perindopril, alone or in combination with indapamide, showed evidence of reverse remodeling of the coronary microvasculature, paralleled by an increased coronary flow, yielding an inverse relationship between hyperemic coronary flow and 
arteriolar medial area [9]. The improved spatial resolution of the latest generation of 3D PET scanners [55] has allowed the quantification of the transmural distribution of MBF in patients with LV hypertrophy secondary to aortic stenosis, demonstrating a more significant reduction of subendocardial CFR, which was directly related to the reduction of the aortic valve area [56].

\section{Non-nuclear techniques: cardiovascular/cardiac magnetic resonance}

Assessment of myocardial perfusion by means of CMR exploits the first pass kinetics of T1-enhancing extra-cellular gadolinium chelates. During the first pass, the contrast medium diffuses in the interstitial space from the microvasculature resulting in increased signal intensity, proportional to the perfusion and blood volume of the tissue, extravascular compartment size, and capillary permeability [57]. Gadolinium chelates do not cross intact cell membranes and the volume of distribution is restricted to approximately $20 \%$ in normal viable myocardium [58]. Different extravascular, extracellular contrast medium doses, administration protocols and pulse sequences have been tested and validated in animal experiments [59-61] which have subsequently been translated into human studies [62-65]. The main issue in the design of CMR perfusion pulse sequences is the trade-off between spatial and temporal resolution, linearity between signal intensity and contrast agent concentration, acquisition time and signal-to-noise ratio. Over time, different solutions to the above problems have been proposed by several groups to optimize performance [66]. The need for standardization of protocols and of image artifacts is a factor limiting clinical performance. The time signal intensity curves may be degraded and this prevents accurate analysis. The worst artifacts are subendocardial dark rims, which can be visually misdiagnosed as perfusion defects.

In everyday clinical practice, qualitative analysis consisting of visual examination of the homogeneous increase in signal during the first pass followed by contrast washout is generally employed. The detection of a delayed signal increase and persistently hypointense regions indicates that the blood supply is reduced by a high-grade stenosis [67, 68]. Interpretation of the data can be semi-quantitative, using the signal intensity index and/or the up-slope rate $[65,69]$, or fully quantitative $[70,71]$. Fully quantitative perfusion analysis is not yet widely used in clinical practice and is performed in highly specialized centers, either calculating various parameters of the tissue enhancement curves and correcting them by subtracting or scaling the arterial input function derived from the LV [59, 65] or using a model-independent deconvolution [72].
Two main approaches are currently under consideration for widespread clinical application: a hyperemic stress only protocol and a stress-rest protocol for the assessment of CFR. The stress-rest protocol offers the advantage of improving the discrimination of dark rim artifacts from truly impaired perfusion in regions without prior myocardial infarction. However, the issue of residual ischemia in an infarcted region remains incompletely addressed [67].

There is accruing evidence of the diagnostic performance of CMR stress perfusion with adenosine in patients with overt CAD (stenosis $>50 \%$ ) and in patients with a lower likelihood of disease or risk factors. A multicenter [73] study has shown good sensitivity (range 90-84 \%) and specificity (range $89-58 \%$ ) for CAD detection on a perpatient basis in comparison with conventional coronary X-ray angiography. In the Multi-Ethnic Study of Atherosclerosis (MESA), a prospective study designed to evaluate risk factors and progression of cardiovascular disease, myocardial perfusion was assessed in 222 subjects [74]. In 28 subjects, the reproducibility and repeatability over an average of 1 year were comparable with PET with relative repeatability coefficients of $30 \%$ of mean at rest and $41 \%$ during hyperemia in the whole heart $[75,76]$.

In 18 centers in Europe and the USA, the diagnostic performance of CMR perfusion assessed qualitatively has been compared head to head, for the detection of CAD, with more established non-invasive techniques such as SPECT using conventional coronary angiography as the standard of reference. CMR perfusion proved equivalent to gated SPECT with $85 \%$ sensitivity and $67 \%$ specificity, and the receiver-operator characteristic curve analysis favoured CMR perfusion over gated and non-gated SPECT [73]. A recent meta-analysis confirmed the diagnostic performance of stress myocardial perfusion CMR in the detection of significant CAD [77]. The data were derived from studies employing 1.5 Tesla magnets and invasive coronary angiography as the reference standard for diagnosing significant obstructive CAD. Hamon and colleagues [77] combined data from more than 11,000 patients in 26 different studies to demonstrate an overall patient-based sensitivity of 0.89 and specificity of 0.80 for the detection of CAD. Out of 26 studies, 20 were evaluated qualitatively and six semi-quantitatively. It has to be noted that high heart rate, motion artifacts due to breathing and low differences in contrast enhancement between normal and underperfused myocardium represented a limitation in the interpretation of the images. Scanners with higher magnetic field strength (e.g., $3 \mathrm{~T}$ ) have increased signal-to-noise ratio and contrast enhancement, which can be used to improve spatial resolution and image quality [78].

In summary, PET has been shown to provide accurate and reproducible measurements of MBF and the availability of non-cyclotron-dependent tracers might increase 
its clinical use. CMR, on the other hand, is more readily available and has lower costs, although MBF quantification is not yet fully validated.

\section{Conclusions}

Pathophysiological mechanisms, assessments, clinical relevance and therapeutic implications vary in different types of CMD [5, 19]. CMD can be the functional counterpart of traditional coronary risk factors. In this setting, CMD can be assessed by non-invasive measurement of CRF and a number of studies have demonstrated that PET is the ideal technique for measuring MBF and CFR, even though its clinical application continues to be rather limited in Europe for a number of reasons, including tracer availability, costs and awareness among referring clinicians. As this type of CMD is, at least in part, reversible, its assessment might be used to guide interventions aimed at reducing risk factor burden. Likewise, PET has been the main technique used to investigate CMD in cardiomyopathies. In hypertrophic and dilated cardiomyopathies, MBF and CFR measured by PET can be useful in risk stratification.

In CAD patients, CMD should be suspected in cases whose symptoms are worse than anticipated on the basis of the severity and extent of angiographic findings. In these patients, CMD might become a new therapeutic target. CMD can be assessed invasively by measuring TIMI frame count and TIMI blush grade, while CFR can be assessed invasively by intracoronary flow wires and non-invasively by contrast echocardiography, PET or CMR.

Conflict of interest Paolo G. Camici: no conflict to declare. Ornella Rimoldi: no conflict to declare.

Human and Animal Studies This article does not contain any studies with human or animal subjects performed by any of the authors.

\section{References}

1. Mueller RL, Sanborn TA (1995) The history of interventional cardiology: cardiac catheterization, angioplasty, and related interventions. Am Heart J 129:146-172. doi:10.1016/00028703(95)90055-1

2. Schwartzkopff B, Frenzel H, Dieckerhoff J, Betz P, Flasshove M, Schulte HD et al (1992) Morphometric investigation of human myocardium in arterial hypertension and valvular aortic stenosis. Eur Heart J 13(Suppl D):17-23

3. Favaloro RG, Effler DB, Groves LK, Sheldon WC, Shirey EK, Sones FM Jr (1970) Severe segmental obstruction of the left main coronary artery and its divisions. Surgical treatment by the saphenous vein graft technique. J Thorac Cardiovasc Surg 60:469-482
4. Gruntzig A, Schneider HJ (1977) The percutaneous dilatation of chronic coronary stenoses-experiments and morphology. Schweiz Med Wochenschr 107:1588

5. Camici PG, Crea F (2007) Coronary microvascular dysfunction. N Engl J Med 356:830-840

6. Maron BJ, Wolfson JK, Epstein SE, Roberts WC (1986) Intramural ("small vessel") coronary artery disease in hypertrophic cardiomyopathy. J Am Coll Cardiol 8:545-557

7. Camici PG, Olivotto I, Rimoldi OE (2012) The coronary circulation and blood flow in left ventricular hypertrophy. J Mol Cell Cardiol 52:857-864. doi:10.1016/j.yjmcc.2011.08.028

8. Mancini M, Petretto E, Kleinert C, Scavone A, De T, Cook S et al (2013) Mapping genetic determinants of coronary microvascular remodeling in the spontaneously hypertensive rat. Basic Res Cardiol 108:316. doi:10.1007/s00395-012-0316-y

9. Neglia D, Fommei E, Varela-Carver A, Mancini M, Ghione S, Lombardi $\mathrm{M}$ et al (2011) Perindopril and indapamide reverse coronary microvascular remodelling and improve flow in arterial hypertension. J Hypertens 29:364-372. doi:10.1097/HJH.0b013 e328340a08e

10. Yoshinaga K, Manabe O, Tamaki N (2011) Assessment of coronary endothelial function using PET. J Nucl Cardiol. 18:486-500. doi:10.1007/s12350-011-9370-9373

11. Crea F, Lanza G, Camici PG (2014) Coronary microvascular dysfunction. Springer, Milan (in press)

12. Inoue K, Hamada M, Ohtsuka T, Hara Y, Shigematsu Y, Nakata $S$ et al (2004) Myocardial microvascular abnormalities observed by intravenous myocardial contrast echocardiography in patients with hypertrophic cardiomyopathy. Am J Cardiol 94:55-58. doi:10.1016/j.amjcard.2004.03.030

13. Cannon RO III, Rosing DR, Maron BJ, Leon MB, Bonow RO, Watson RM et al (1985) Myocardial ischemia in patients with hypertrophic cardiomyopathy: contribution of inadequate vasodilator reserve and elevated left ventricular filling pressures. Circulation 71:234-243

14. Heusch G (2010) Adenosine and maximum coronary vasodilation in humans: myth and misconceptions in the assessment of coronary reserve. Basic Res Cardiol 105:1-5. doi:10.1007/s00395009-0074-7

15. Kaufmann PA, Camici PG (2005) Myocardial blood flow measurement by PET: technical aspects and clinical applications. J Nucl Med 46:75-88

16. Chareonthaitawee P, Kaufmann PA, Rimoldi O, Camici PG (2001) Heterogeneity of resting and hyperemic myocardial blood flow in healthy humans. Cardiovasc Res 50:151-161. doi:S0008 636301002024

17. Rechavia E, Araujo LI, de Silva R, Kushwaha SS, Lammertsma AA, Jones T et al (1992) Dipyridamole vasodilator response after human orthotopic heart transplantation: Quantification by oxygen-15-labeled water and positron emission tomography. J Am Coll Cardiol 19:100-106. doi:10.1016/0735-1097(92)90058-U

18. Uren NG, Melin JA, De Bruyne B, Wijins W, Baudhuin T, Camici PG (1994) Relation between myocardial blood flow and the severity of coronary artery stenosis. N Engl J Med 330:1782-1788

19. Camici PG, Rimoldi OE (2009) The clinical value of myocardial blood flow measurement. J Nucl Med 50:1076-1087. doi:10. 2967/jnumed.108.054478

20. Hajjiri MM, Leavitt MB, Zheng H, Spooner AE, Fischman AJ, Gewirtz H (2009) Comparison of positron emission tomography measurement of adenosine-stimulated absolute myocardial blood flow versus relative myocardial tracer content for physiological assessment of coronary artery stenosis severity and location. JACC Cardiovasc Imaging 2:751-758. doi:10.1016/j.jcmg.2009.04.004

21. Herzog BA, Husmann L, Valenta I, Gaemperli O, Siegrist PT, Tay FM et al (2009) Long-term prognostic value of 
$13 \mathrm{~N}$-ammonia myocardial perfusion positron emission tomography added value of coronary flow reserve. J Am Coll Cardiol 54:150-156. doi:10.1016/j.jacc.2009.02.069

22. Cecchi F, Olivotto I, Gistri R, Lorenzoni R, Chiriatti G, Camici PG (2003) Coronary microvascular dysfunction and prognosis in hypertrophic cardiomyopathy. N Engl J Med 349:1027-1035

23. Neglia D, Michelassi C, Trivieri MG, Sambuceti G, Giorgetti A, Pratali L et al (2002) Prognostic role of myocardial blood flow impairment in idiopathic left ventricular dysfunction. Circulation 105:186-193

24. Di Carli MF, Janisse J, Grunberger G, Ager J (2003) Role of chronic hyperglycemia in the pathogenesis of coronary microvascular dysfunction in diabetes. $\mathrm{J}$ Am Coll Cardiol 41:1387-1393

25. Bergmann S, Herrero P, Markham J, Winheimer C, Walsh M (1989) Noninvasive quantification of myocardial blood flow in human subjects with oxygen-15-labeled water and positron emission tomography. J Am Coll Cardiol 14:639-652

26. Araujo LI, Lammerstma AA, Rhodes CG, McFalls EO, Lida H, Rechavia $\mathrm{E}$ et al (1991) Noninvasive quantification of regional myocardial blood flow in normal volunteers and patients with coronary artery disease using oxygen- 15 labeled water and positron emission tomography. Circulation 83:875-885

27. Kaufmann P, Gnecchi-Ruscone T, Yap J, Rimoldi O, Camici P (1999) Assessment of the reproducibility of baseline and hyperemic myocardial blood flow measurements with 15-O-labeled water and PET. J Nucl Med 40:1848-1856

28. Schelbert HR, Phelps ME, Hoffman EJ, Huang SC, Selin CE, Kuhl DE (1979) Regional myocardial perfusion assessed with $\mathrm{N}-13$ labeled ammonia and positron emission computerized axial tomography. Am J Cardiol 43:209-218

29. Hutchins G, Schwaiger M, Rosenspire KC, Krivokapich J, Schelbert H, Kuhl D (1990) Noninvasive quantification of regional blood flow in the human heart using N-13 ammonia and dynamic positron emission tomographic imaging. J Am Coll Cardiol 15:1032-1042

30. Krivokapich J, Smith GT, Huang SC, Hoffman EJ, Ratib O, Phelps ME et al (1989) $13 \mathrm{~N}$ ammonia myocardial imaging at rest and with exercise in normal volunteers. Quantification of absolute myocardial perfusion with dynamic positron emission tomography. Circulation 80:1328-1337

31. Muzik O, Beanlands RS, Hutchins GD, Mangner TJ, Nguyen N, Schwaiger M (1993) Validation of nitrogen-13-ammonia tracer kinetic model for quantification of myocardial blood flow using PET. J Nucl Med 34:83-91

32. Herrero P, Markham J, Shelton ME, Weinheimer CJ, Bergmann SR (1990) Noninvasive quantification of regional myocardial perfusion with rubidium-82 and positron emission tomography. Exploration of a mathematical model. Circulation 82:1377-1386

33. El Fakhri G, Kardan A, Sitek A, Dorbala S, Abi-Hatem N, Lahoud Y et al (2009) Reproducibility and accuracy of quantitative myocardial blood flow assessment with (82)Rb PET: comparison with (13)N-ammonia PET. J Nucl Med 50:1062-1071. doi:10. 2967/jnumed.104.007831

34. Bergmann SR, Weinheimer CJ, Brown MA, Perez JE (1994) Enhancement of regional myocardial efficiency and persistence of perfusion, oxidative, and functional reserve with paired pacing of stunned myocardium. Circulation 89:2290-2296

35. Iida H, Kanno I, Takahashi A, Miura S, Murakami M, Takahashi $\mathrm{K}$ et al (1988) Measurement of absolute myocardial blood flow with $\mathrm{H} 215 \mathrm{O}$ and dynamic positron-emission tomography. Strategy for quantification in relation to the partial-volume effect. Circulation 78:104-115

36. Hermansen F, Rosen SD, Fath-Ordoubadi F, Kooner JS, Clark JC, Camici PG et al (1998) Measurement of myocardial blood flow with oxygen-15 labelled water: comparison of different administration protocols. Eur J Nucl Med 25:751-759

37. Huang SC, Schwaiger M, Carson RE, Carson J, Hansen H, Selin C et al (1985) Quantitative measurement of myocardial blood flow with oxygen-15 water and positron computed tomography: an assessment of potential and problems. J Nucl Med 26:616-625

38. Anagnostopoulos C, Almonacid A, El Fakhri G, Curillova Z, Sitek A, Roughton M et al (2008) Quantitative relationship between coronary vasodilator reserve assessed by $82 \mathrm{Rb}$ PET imaging and coronary artery stenosis severity. Eur J Nucl Med Mol Imaging 35:1593-1601

39. Lautamaki R, George RT, Kitagawa K, Higuchi T, Merrill J, Voicu C et al (2009) Rubidium-82 PET-CT for quantitative assessment of myocardial blood flow: validation in a canine model of coronary artery stenosis. Eur J Nucl Med Mol Imaging 36:576-586. doi:10.1007/s00259-008-0972-1

40. Lortie M, Beanlands RS, Yoshinaga K, Klein R, Dasilva JN, DeKemp RA (2007) Quantification of myocardial blood flow with $82 \mathrm{Rb}$ dynamic PET imaging. Eur J Nucl Med Mol Imaging 34:1765-1774

41. Dorbala S, Di Carli MF, Beanlands RS, Merhige ME, Williams BA, Veledar E et al (2013) Prognostic value of stress myocardial perfusion positron emission tomography: results from a multicenter observational registry. J Am Coll Cardiol 61:176-184. doi:10.1016/j.jacc.2012.09.043

42. Murthy VL, Naya M, Foster CR, Hainer J, Gaber M, Di Carli G et al (2011) Improved cardiac risk assessment with noninvasive measures of coronary flow reserve. Circulation 124:2215-2224. doi:10.1161/CIRCULATIONAHA.111.050427

43. Nagamachi S, Czernin J, Kim AS, Sun KT, Bottcher M, Phelps ME et al (1996) Reproducibility of measurements of regional resting and hyperemic myocardial blood flow assessed with PET. J Nucl Med 37:1626-1631

44. Efseaff M, Klein R, Ziadi MC, Beanlands RS, deKemp RA (2012) Short-term repeatability of resting myocardial blood flow measurements using rubidium-82 PET imaging. J Nucl Cardiol 19:997-1006. doi:10.1007/s12350-012-9600-3

45. Wyss CA, Koepfli P, Mikolajczyk K, Burger C, von Schulthess GK, Kaufmann PA (2003) Bicycle exercise stress in PET for assessment of coronary flow reserve: repeatability and comparison with adenosine stress. J Nucl Med 44:146-154

46. Jagathesan R, Kaufmann PA, Rosen SD, Rimoldi OE, Turkeimer F, Foale R et al (2005) Assessment of the long-term reproducibility of baseline and dobutamine-induced myocardial blood flow in patients with stable coronary artery disease. J Nucl Med 46:212-219

47. Danad I, Raijmakers PG, Appelman YE, Harms HJ, de Haan S, van den Oever ML et al (2013) Hybrid imaging using quantitative H215O PET and CT-based coronary angiography for the detection of coronary artery disease. J Nucl Med 54:55-63. doi:10. 2967/jnumed.112.104687

48. Naya M, Murthy VL, Foster CR, Gaber M, Klein J, Hainer J et al (2013) Prognostic interplay of coronary artery calcification and underlying vascular dysfunction in patients with suspected coronary artery disease. J Am Coll Cardiol 61:2098-2106. doi:10. 1016/j.jacc.2013.02.029

49. Ziadi MC, Dekemp RA, Williams K, Guo A, Renaud JM, Chow BJ et al (2012) Does quantification of myocardial flow reserve using rubidium-82 positron emission tomography facilitate detection of multivessel coronary artery disease? J Nucl Cardiol 19:670-680. doi:10.1007/s12350-011-9506-5

50. Basso C, Thiene G, Corrado D, Buja G, Melacini P, Nava A (2000) Hypertrophic cardiomyopathy and sudden death in the young: pathologic evidence of myocardial ischemia. Hum Pathol 31:988-998. doi:10.1053/hupa.2000.16659 
51. Maron MS, Olivotto I, Maron BJ, Prasad SK, Cecchi F, Udelson JE et al (2009) The case for myocardial ischemia in hypertrophic cardiomyopathy. J Am Coll Cardiol 54:866-875. doi:10.1016/j. jacc.2009.04.072

52. Tomanek RJ (1990) Response of the coronary vasculature to myocardial hypertrophy. J Am Coll Cardiol 15:528-533. doi:10. 1016/0735-1097(90)90620-5

53. Hamasaki S, Al Suwaidi J, Higano ST, Miyauchi K, Holmes DR Jr, Lerman A (2000) Attenuated coronary flow reserve and vascular remodeling in patients with hypertension and left ventricular hypertrophy. J Am Coll Cardiol 35:1654-1660. doi:10.1016/ s0735-1097(00)00594-5

54. Schindler TH, Nitzsche EU, Olschewski M, Brink I, Mix M, Prior $\mathrm{J}$ et al (2004) PET-measured responses of MBF to cold pressor testing correlate with indices of coronary vasomotion on quantitative coronary angiography. J Nucl Med 45:419-428

55. Rimoldi O, Schafers KP, Boellaard R, Turkheimer F, Stegger L, Law MP et al (2006) Quantification of subendocardial and subepicardial blood flow using 15O-labeled water and PET: experimental validation. J Nucl Med 47:163-172

56. Rajappan K, Rimoldi O, Camici PG, Pennell DJ, Sheridan DJ (2002) Factors influencing coronary microcirculatory function in patients with aortic stenosis after aortic valve replacement. Circulation 106:640-641

57. Tong CY, Prato FS, Wisenberg G, Lee TY, Carroll E, Sandler D et al (1993) Measurement of the extraction efficiency and distribution volume for Gd-DTPA in normal and diseased canine myocardium. Magn Reson Med 30:337-346

58. Schwitter J, Saeed M, Wendland MF, Derugin N, Canet E, Brasch RC et al (1997) Influence of severity of myocardial injury on distribution of macromolecules: extravascular versus intravascular gadolinium-based magnetic resonance contrast agents. J Am Coll Cardiol 30:1086-1094

59. Lee DC, Simonetti OP, Harris KR, Holly TA, Judd RM, Wu E et al (2004) Magnetic resonance versus radionuclide pharmacological stress perfusion imaging for flow-limiting stenoses of varying severity. Circulation 110:58-65

60. Goldstein TA, Jerosch-Herold M, Misselwitz B, Zhang H, Gropler RJ, Zheng J (2008) Fast mapping of myocardial blood flow with MR first-pass perfusion imaging. Magn Reson Med 59:1394-1400

61. Christian TF, Aletras AH, Arai AE (2008) Estimation of absolute myocardial blood flow during first-pass MR perfusion imaging using a dual-bolus injection technique: comparison to singlebolus injection method. J Magn Reson Imaging 27:1271-1277. doi:10.1002/jmri.21383

62. Cheng AS, Pegg TJ, Karamitsos TD, Searle N, Jerosch-Herold M, Choudhury RP et al (2007) Cardiovascular magnetic resonance perfusion imaging at 3-tesla for the detection of coronary artery disease: a comparison with 1.5-tesla. J Am Coll Cardiol 49:2440-2449

63. Hsu LY, Rhoads KL, Holly JE, Kellman P, Aletras AH, Arai AE (2006) Quantitative myocardial perfusion analysis with a dualbolus contrast-enhanced first-pass MRI technique in humans. J Magn Reson Imaging 23:315-322

64. Petersen SE, Jerosch-Herold M, Hudsmith LE, Robson MD, Francis JM, Doll HA et al (2007) Evidence for microvascular dysfunction in hypertrophic cardiomyopathy: new insights from multiparametric magnetic resonance imaging. Circulation 115:2418-2425

65. Schwitter J, Nanz D, Kneifel S, Bertschinger K, Buchi M, Knusel PR et al (2001) Assessment of myocardial perfusion in coronary artery disease by magnetic resonance: a comparison with positron emission tomography and coronary angiography. Circulation 103:2230-2235

66. Kellman P, Arai AE (2007) Imaging sequences for first pass perfusion-a review. J Cardiovasc Magn Reson 9:525-537

67. Klem I, Heitner JF, Shah DJ, Sketch MH Jr, Behar V, Weinsaft J et al (2006) Improved detection of coronary artery disease by stress perfusion cardiovascular magnetic resonance with the use of delayed enhancement infarction imaging. J Am Coll Cardiol 47:1630-1638

68. Ingkanisorn WP, Kwong RY, Bohme NS, Geller NL, Rhoads KL, Dyke CK et al (2006) Prognosis of negative adenosine stress magnetic resonance in patients presenting to an emergency department with chest pain. J Am Coll Cardiol 47:1427-1432

69. Al-Saadi N, Nagel E, Gross M, Bornstedt A, Schnackenburg B, Klein $C$ et al (2000) Noninvasive detection of myocardial ischemia from perfusion reserve based on cardiovascular magnetic resonance. Circulation 101:1379-1383

70. Larghat A, Biglands J, Maredia N, Greenwood JP, Ball SG, Jerosch-Herold $\mathrm{M}$ et al (2012) Endocardial and epicardial myocardial perfusion determined by semi-quantitative and quantitative myocardial perfusion magnetic resonance. Int $\mathrm{J}$ Cardiovasc Imaging 28:1499-1511. doi:10.1007/s10554-011-9982-3

71. Coelho-Filho OR, Seabra LF, Mongeon FP, Abdullah SM, Francis SA, Blankstein R et al (2011) Stress myocardial perfusion imaging by $\mathrm{CMR}$ provides strong prognostic value to cardiac events regardless of patient's sex. JACC Cardiovascular imaging 4:850-861. doi:10.1016/j.jcmg.2011.04.015

72. Jerosch-Herold M, Swingen C, Seethamraju RT (2002) Myocardial blood flow quantification with MRI by model-independent deconvolution. Med Phys 29:886-897

73. Schwitter J, Wacker CM, van Rossum AC, Lombardi M, AlSaadi N, Ahlstrom H et al (2008) MR-IMPACT: comparison of perfusion-cardiac magnetic resonance with single-photon emission computed tomography for the detection of coronary artery disease in a multicentre, multivendor, randomized trial. Eur Heart J 29:480-489. doi:10.1093/eurheartj/ehm617

74. Wang L, Jerosch-Herold M, Jacobs JDR, Shahar E, Folsom AR (2006) Coronary risk factors and myocardial perfusion in asymptomatic adults: the Multi-Ethnic Study of Atherosclerosis (MESA). J Am Coll Cardiol 47:565-572

75. Jagathesan R, Barnes E, Rosen SD, Foale RA, Camici PG (2006) Comparison of myocardial blood flow and coronary flow reserve during dobutamine and adenosine stress: implications for pharmacologic stress testing in coronary artery disease. J Nucl Cardiol 13:324-332

76. Jerosch-Herold M, Vazquez G, Wang L, Jacobs DR Jr, Folsom AR (2008) Variability of myocardial blood flow measurements by magnetic resonance imaging in the multi-ethnic study of atherosclerosis. Invest Radiol 43:155-161

77. Hamon M, Fau G, Nee G, Ehtisham J, Morello R (2010) Metaanalysis of the diagnostic performance of stress perfusion cardiovascular magnetic resonance for detection of coronary artery disease. J Cardiovasc Magn Reson 12:29. doi:10.1186/1532429X-12-29

78. Lockie T, Ishida M, Perera D, Chiribiri A, De Silva K, Kozerke S et al (2011) High-resolution magnetic resonance myocardial perfusion imaging at 3.0-Tesla to detect hemodynamically significant coronary stenoses as determined by fractional flow reserve. J Am Coll Cardiol 57:70-75. doi:10.1016/j.jacc.2010.09.019 